\title{
Comparing the low-temperature performance of megapixel NIR InGaAs and HgCdTe imager arrays
}

S. Seshadri, D. M. Cole, B. Hancock, P. Ringold, C. Peay, et al.

S. Seshadri, D. M. Cole, B. Hancock, P. Ringold, C. Peay, C. Wrigley, M. Bonati, M. G. Brown, M. Schubnell, G. Rahmer, D. Guzman, D. Figer, G. Tarle, R. M. Smith, C. Bebek, "Comparing the low-temperature performance of megapixel NIR InGaAs and HgCdTe imager arrays," Proc. SPIE 6690, Focal Plane Arrays for Space Telescopes III, 669006 (14 September 2007); doi: $10.1117 / 12.734739$

Event: Optical Engineering + Applications, 2007, San Diego, California, United States 


\title{
Comparing the low-temperature performance of megapixel NIR InGaAs and $\mathrm{HgCdTe}$ imager arrays
}

\author{
S. Seshadri ${ }^{\mathrm{a}}$, D.M. Cole ${ }^{\mathrm{a}}$, B. Hancock ${ }^{\mathrm{a}}$, P. Ringold ${ }^{\mathrm{a}}$, C. Peay ${ }^{\mathrm{a}}$, C. Wrigley $^{\mathrm{a}}$, M. Bonati $^{\mathrm{b}}$, M.G. Brown $^{\mathrm{c}}$, \\ M. Schubnell ${ }^{\mathrm{c}}$, G. Rahmer ${ }^{\mathrm{b}}$, D. Guzman ${ }^{\mathrm{b}}$, D. Figer $^{\mathrm{d}}$, G. Tarle ${ }^{\mathrm{c}}$, R.M. Smith ${ }^{\mathrm{b}}$, and C. Bebek ${ }^{\mathrm{e}}$ \\ ${ }^{\mathrm{a}}$ Jet Propulsion Laboratory, ${ }^{\mathrm{b}}$ California Institute of Technology, Pasadena, CA, ${ }^{\mathrm{c} U n i v e r s i t y ~ o f ~ M i c h i g a n, ~ A n n ~ A r b o r, ~ M I, ~}$ \\ ${ }^{\mathrm{d}}$ Rochester Institute of Technology, Rochester, NY and ${ }^{\mathrm{e}}$ Lawrence Berkeley National Laboratory, Berkeley, CA, \\ Department of Physics \\ MS 300-315, Jet Propulsion Laboratory, 4800 Oak Grove Drive, Pasadena, CA, USA 91109, Email: \\ suresh.seshadri@jpl.nasa.gov; PH: (818)-354-8370
}

\begin{abstract}
We compare a more complete characterization of the low temperature performance of a nominal 1.7um cut-off wavelength $1 \mathrm{kx} 1 \mathrm{k}$ InGaAs (lattice-matched to an InP substrate) photodiode array against similar, $2 \mathrm{kx} 2 \mathrm{k} \mathrm{HgCdTe}$ imagers to assess the suitability of InGaAs FPA technology for scientific imaging applications. The data we present indicate that the low temperature performance of existing InGaAs detector technology is well behaved and comparable to those obtained for state-of-the-art $\mathrm{HgCdTe}$ imagers for many space astronomical applications. We also discuss key differences observed between imagers in the two material systems.
\end{abstract}

Keywords: near-infrared, InGaAs, $\mathrm{HgCdTe}$, low-temperature, detector, imager, focal plane array, science

\section{INTRODUCTION}

Future astrophysics missions will require detector-limited science performance for large (100s) Near Infra-Red (NIR) focal planes. While we have seen significant improvements in the performance of existing 1.7 um cut-off $\mathrm{HgCdTe}$ (HCT) focal planes in recent years that have abated some of the concerns about the viability of this technology for the most stringent science applications, ${ }^{1,2}$ these detectors can cost upwards of 8-10 times more than a comparably sized visible CCD. Concerns about the cost/performance ratio for these missions leads one to question whether there is a commercially viable, technological alternative to the HCT focal plane technology that can provide equivalent or better science performance and one that can do it for a lower cost/Mpixel with good reliability.

As Figure 1 illustrates, HCT imagers are favored for the NIR because the tight lattice match of the active detector material to the CdZnTe substrate over the entire HCT composition range enables high performance imagers over a wide wavelength band. Also evident in the figure is that InGaAs, lattice-matched to an InP substrate offers a viable alternative for defect-free, and therefore, potentially imagers with simultaneously high quantum efficiency and low dark current. The long wavelength cut-off for InGaAs at room temperature is $\sim 1.7 \mathrm{um}$, making it suitable for NIR applications. Indeed InGaAs detectors and imagers at this composition are presently used in commercial Dense Wavelength Division Multiplexing (DWDM) telecommunications, surveillance, military and aerospace applications. ${ }^{3,4}$ Despite these potential advantages, the use of InGaAs-based arrays for scientific imaging in the near-infrared is minimal ${ }^{5}$ and not widespread. In terms of performance, InGaAs detectors with high, flat QE already exist. ${ }^{5}$ Technologically, a transparent InP substrate and substrate removal technology, such as that used to increase short wavelength performance and minimize cut-off wavelength non-uniformities and cosmic ray effects in $\mathrm{HgCdTe}$, also exists. These advantages offer imagers based on InGaAs technology the potential to be a lower cost, higher reliability alternative to those based on $\mathrm{HgCdTe}$. We have previously described the low temperature performance of InGaAs FPAs. ${ }^{6}$ The work described herein compares more complete characterization of the low temperature performance of an InGaAs-based imager array to similar HCT FPA than presently exists, to directly assess the suitability of this technology for scientific imaging applications.

Focal Plane Arrays for Space Telescopes III, edited by Thomas J. Grycewicz, Cheryl J. Marshall, Penny G. Warren Proc. of SPIE Vol. 6690, 669006, (2007) · 0277-786X/07/\$18 - doi: 10.1117/12.734739 


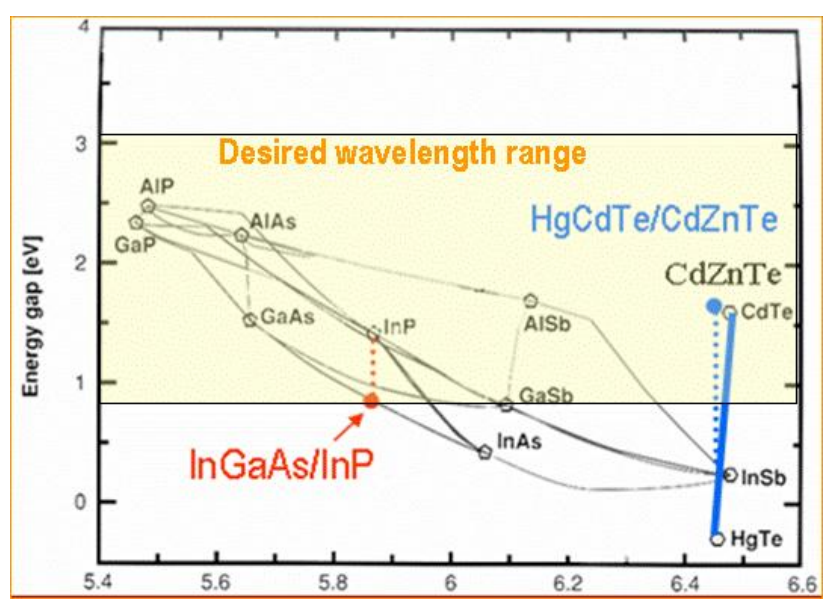

Figure 1: Bandgap energy vs lattice constant plot for different ternary semiconductors.

\section{METHODOLOGY}

We characterized a nominally 1.7 um cut-off wavelength $1 \mathrm{kx} 1 \mathrm{k}$ InGaAs (lattice-matched to an InP substrate) photodiode array fabricated by Sensors Unlimited, Inc. (now part of Goodrich Corporation). The array was hybridized to a Teledyne Imaging Systems (formerly Rockwell Scientific) H1RG multiplexer under the Lawrence Berkeley National Laboratoryled SuperNova/Acceleration Probe (SNAP) mission concept development program. ", ${ }^{1,27}$ The focal plane did not have an anti-reflective coating. The substrate was also not removed. The InGaAs FPA was "run-of-the-mill". The $2 \mathrm{kx} 2 \mathrm{k}, 1.7 \mathrm{um}$ cut-off substrate-removed and non-substrate-removed HCT against which the InGaAs FPAs were compared, were the state-of-the-art.

Comparisons of our InGaAs test data with HCT data are based on results obtained by Roger Smith at the California Institute of Technology, Optical Observatories Department and Professor Greg Tarle's lab at the University of Michigan, Department of Physics under nominally identical conditions: The H1RG multiplexor was was operated in slow readout mode, with buffered output, with an external current source. An array controller from Astronomical Research Cameras, Inc. (commonly referred to as "Generation III, Leach controller") provided clocking and bias signals. Bias levels were generated on an 8-channel analog video processor board. Two of these boards were used for 16 channel readout of the detector. The most important biases settings used in the tests are shown in Table 1. Clocks were provided on a separate digital clock driver board. With the controller driving the multiplexer clock at a rate of $110 \mathrm{kHz}$, the integration time per pixel before conversion was $1 \mu \mathrm{s}$.

The multiplexer was read out in 16-channel output mode, each output corresponding to a 64 column, 1024 row slice of the array. This output was digitized by a 16-bit Analog-toDigital Converter (ADC). The offset of the video board gain stage for each channel was optimized to maximize the $0.5 \mathrm{~V}$ signal swing at the detector over the entire ADC range. Software control of the detector and control electronics was established using custom software. ${ }^{8}$

\begin{tabular}{|l|c|}
\hline \multicolumn{2}{|c|}{$\begin{array}{c}\text { Table 1: H1RG analog } \\
\text { signal chain bias settings. }\end{array}$} \\
\hline Vdd (V) & 3.3 \\
\hline Vbp (V) & 3.3 \\
\hline $\operatorname{Vbg}(\mathrm{V})$ & 2.4 \\
\hline Vdsub (V) & 1.0 \\
\hline Vrst (V) & 0.5 \\
\hline
\end{tabular}

All our data consist either of correlated double sample (CDS) pairs, or of series of samples taken up-the-ramp. In both cases, the detector was reset by clocking through the entire array with reset enabled; the array was then read out immediately, to create a "reset" frame. This corresponds to the H1RG line-by-line reset (option B), which we found was the most effective at repeatably resetting pixels. This minimized data corruption by dynamic self-heating effects in the detector/multiplexer package. For the CDS data, we also clocked through the array without reading it, immediately before the exposure read, to duplicate the initial sample conditions as precisely as possible. Because artifacts were 
sometimes still present, we still routinely removed the first frame from our analysis to minimize any clocking-related issues.

\section{RESULTS}

The mean-variance and conversion gain $\left(\mathrm{C}_{\mathrm{g}}\right)$ for the InGaAs detector are shown in Figures 2 and 3. Note that the engineering definition of $\mathrm{C}_{\mathrm{g}}(\mathrm{uV} / \mathrm{e}-)$ is the inverse of the astronomers' definition. We, therefore, call the latter, the inverse gain $\left(=1 / C_{g}\right)$. From the figure, it is apparent that $C_{g}=10.8 \mathrm{uV} / \mathrm{e}$ - for small signals, but is signal-dependent, with noticeable deviation from constancy at signal levels above $\sim 50 \%$ of full scale. The small-signal $\mathrm{C}_{\mathrm{g}}$ is seen to be $\sim 2 \mathrm{x}$ larger than for the HCT. We have used the method proposed by B. Pain et. al. ${ }^{8}$ to account for the non-linear capacitance of the sense node when calculating $\mathrm{C}_{\mathrm{g}}$ for larger signal levels. The interpixel capacitance was calculated to be $7 \%$ for the InGaAs FPA. ${ }^{9}$ This compares against a value of $\sim 20 \%$ calculated for HCT. ${ }^{10}$ The measured linearity and full well, calculated using the conversion gain data, are illustrated in Figure 4 . The full well for a $0.5 \mathrm{~V}$ detector bias was calculated to be $435 \mathrm{~K}$ e-, much higher than the $\sim 70 \mathrm{Ke}$ - for the HCT, calculated using the small-signal $\mathrm{C}_{\mathrm{g}}$.
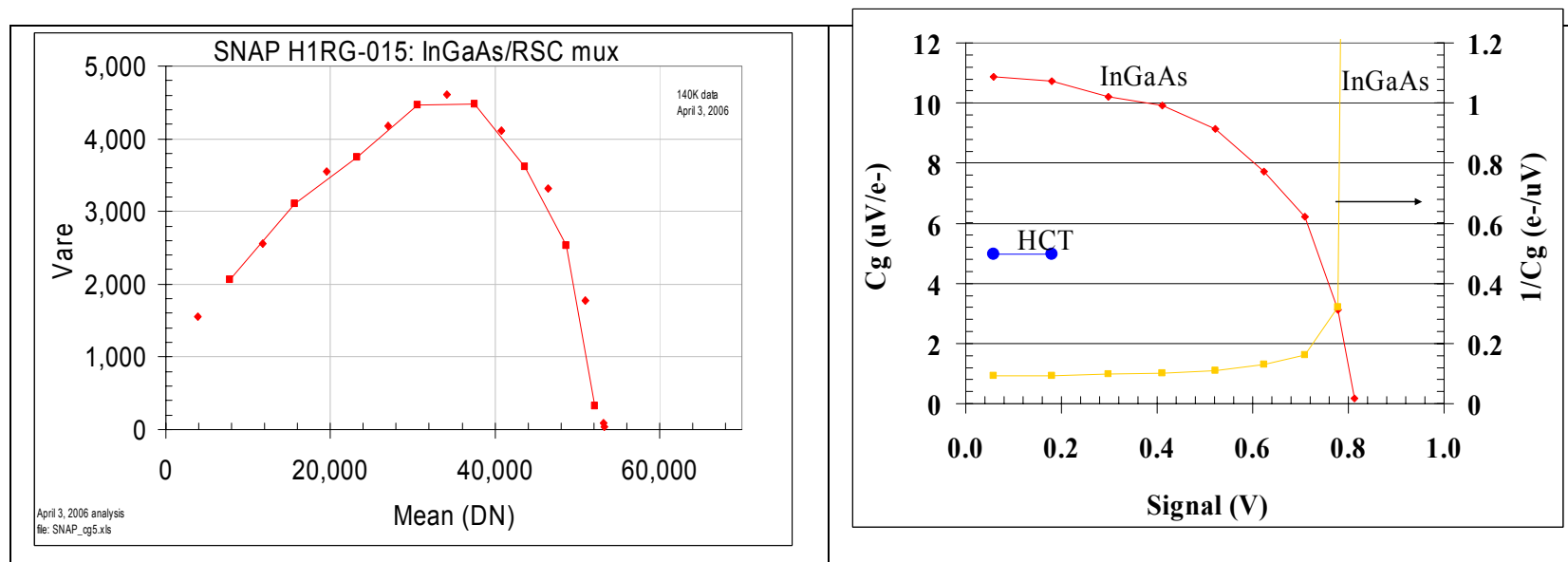

Figure 2: Mean-variance plot for the InGaAs FPA operating at $140 \mathrm{~K}$.

Figure 3: Conversion gain for InGaAs and HCT at 140K.

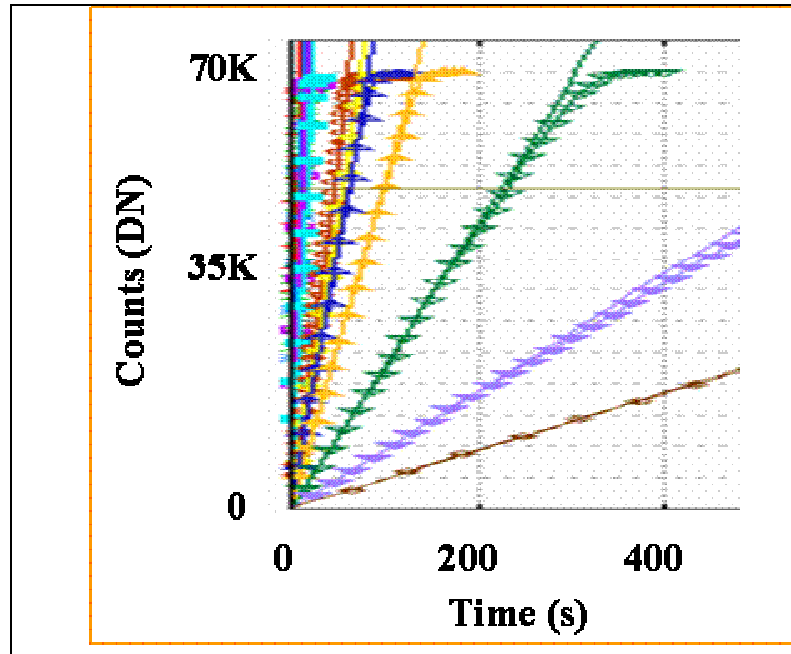

(a)

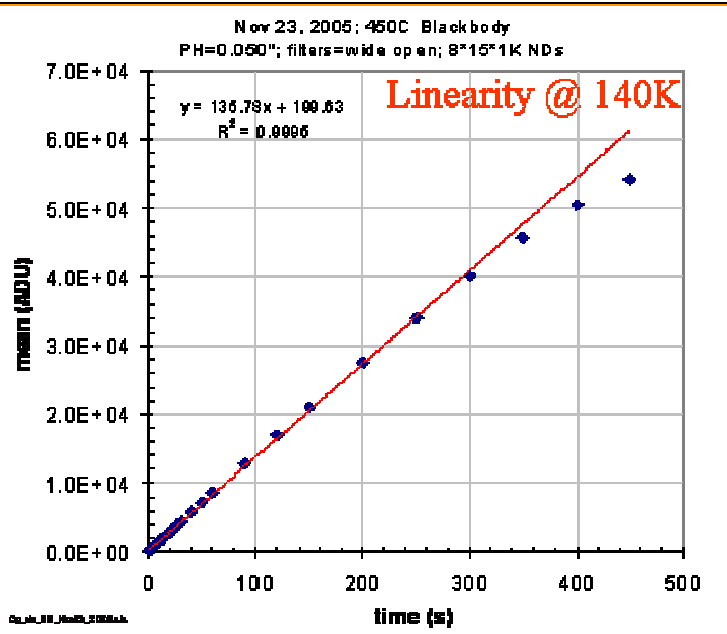

(b)

Figure 4: Comparison of the linearities of (a) HCT and (b) InGaAs. 
Figure 5 illustrates the relative QE of the InGaAs detector at $140 \mathrm{~K}$. The curve has been adjusted to achieve the same maximum absolute $300 \mathrm{~K}$ QE data. A nominal room temperature curve is also shown in the figure for comparison. It is evident that the spectral shape is retained, with the wavelength being shifted towards shorter wavelengths due to the band gap shift of the detector band edge. The shift corresponds to $\sim 80 \mathrm{~nm}$ shorter cut-off wavelength at $140 \mathrm{~K}$ relative to $300 \mathrm{~K}$. This result is a bit higher than the $\sim 100 \mathrm{~nm}$ shift expected from theory, shown in figure $6 .{ }^{11}$ However, the data correlates well with recent results obtained by others. ${ }^{12} \mathrm{We}$ see from figure $5 \mathrm{~b}$ that the InGaAs QE has more ideal spectral shape than the non-substrate-removed HCT FPA, but lower blue response than the substrate-removed HCT. The latter is, of course, expected.

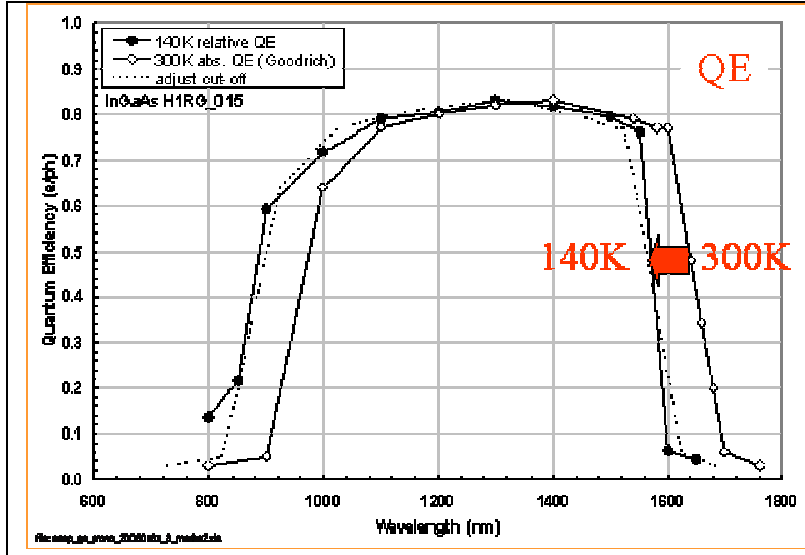

(a)

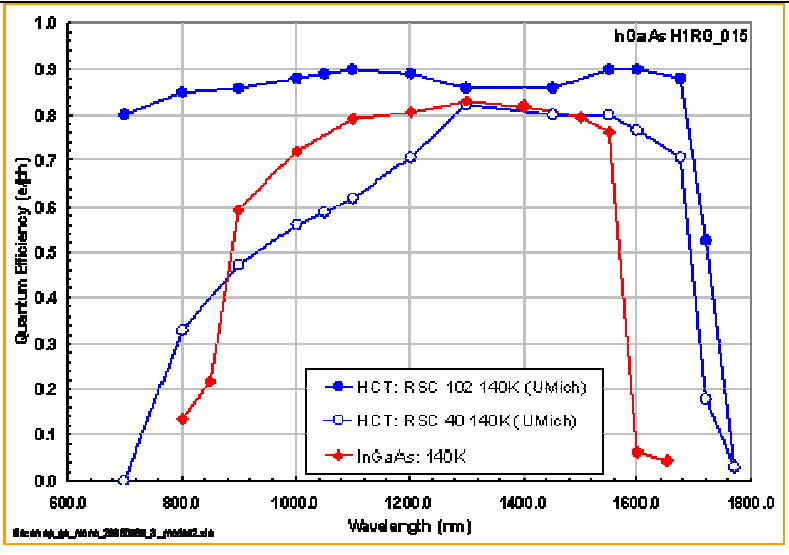

(b)

Figure 5: (a) Variation of QE with temperature for InGaAs. (b) Comparison of QE for InGaAs and non-substrateremoved and substrate-removed HCT. Note that the non-substrate-removed HCT peak QE has been increased by $\sim 15 \%$ to match the InGaAs peak value.

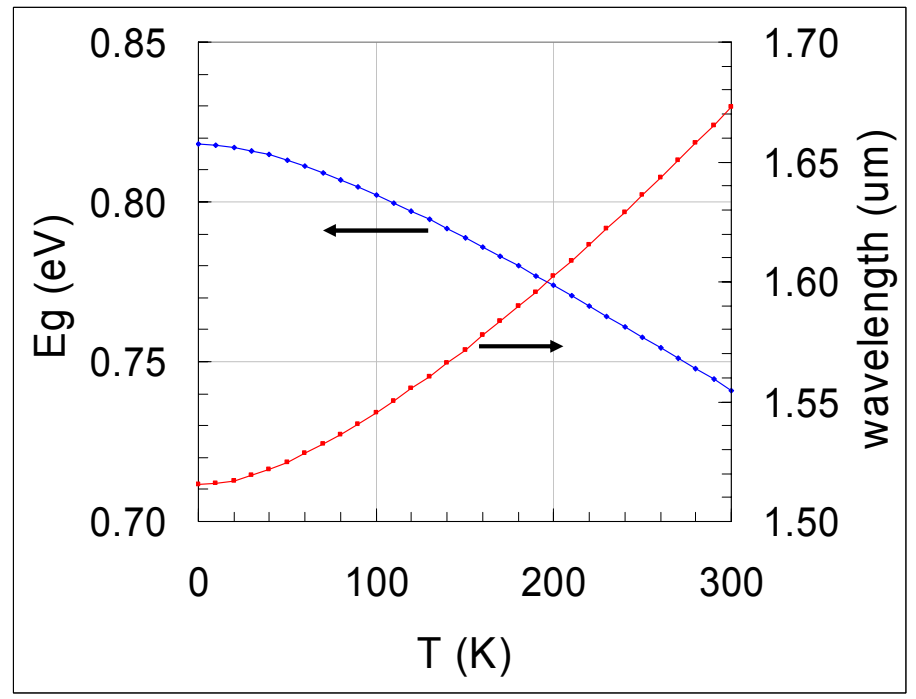

Figure 6: Variation of bandgap energy and long-wavelength cut-off of InGaAs versus temperature.

Figure 7 illustrates histograms of the dark current for the two focal planes at $140 \mathrm{~K}$. While both imagers exhibit the high dark current tail, the InGaAs FPA appears to have a noticeably tighter distribution than the HCT device. Figure 8 
illustrates an Arrhenius plot of the temperature dependence of the mean dark current of InGaAs versus another HCT. The InGaAs dark current is relatively well behaved with an extrapolated value that matches nominal room temperature data. The calculated activation energy of $0.25 \mathrm{eV}$ is a bit lower than the $0.37 \mathrm{eV}$ expected for purely generationrecombination in the depletion region. The flattening of the dark current at low temperatures is thought to be due to the effect of photo-generation from diffuse cosmic rays in the InP substrate, as has been observed for non-substrate-removed HgCdTe. $^{2}$

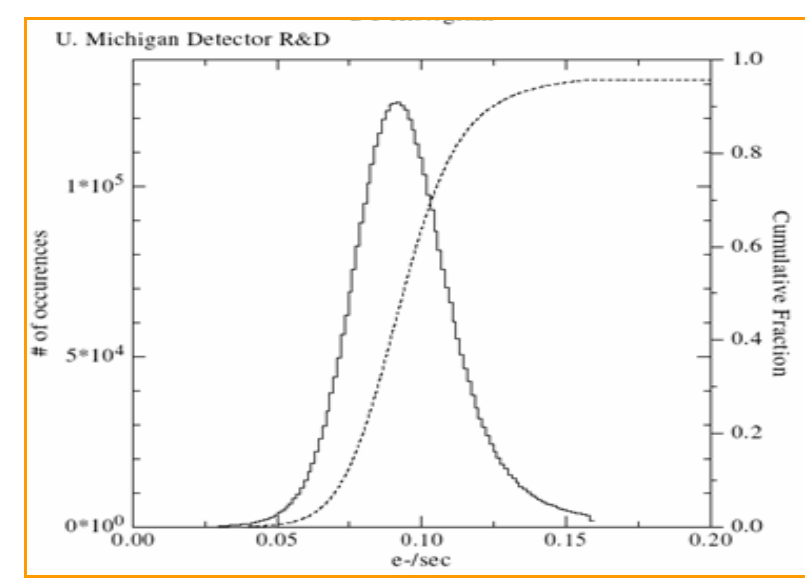

(a)

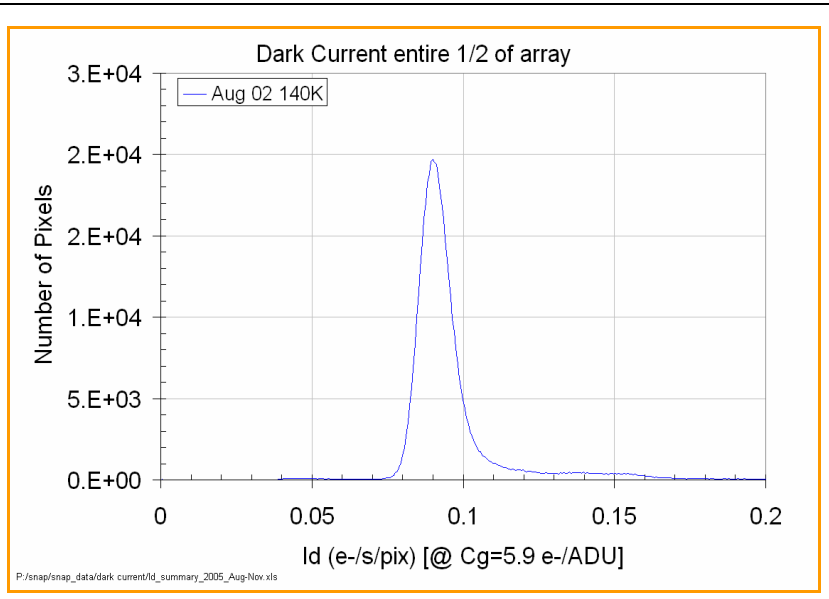

(b)

Figure 7: Comparison of 140K dark currents of (a) HCT and (b) InGaAs.

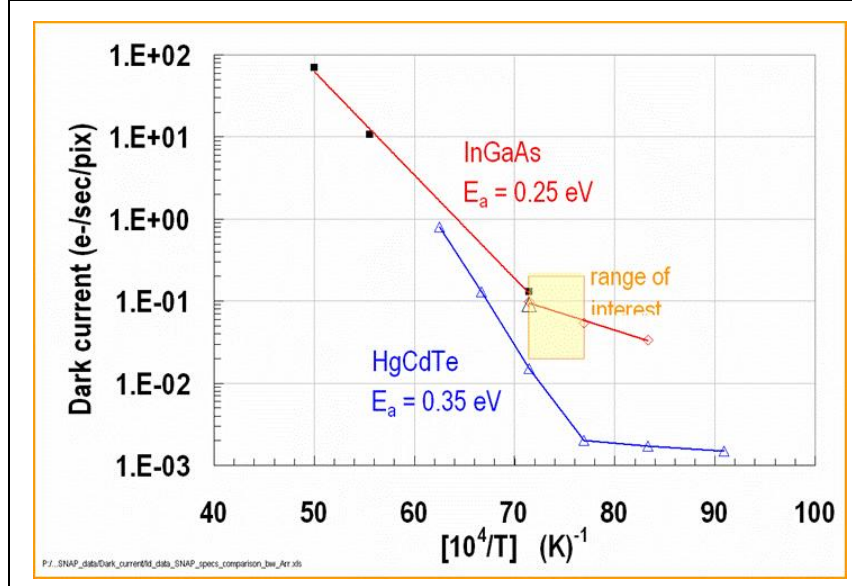

Figure 8: Comparison of the temperature dependence of the dark current of HCT and InGaAs. The HCT FPA has its substrate-removed.

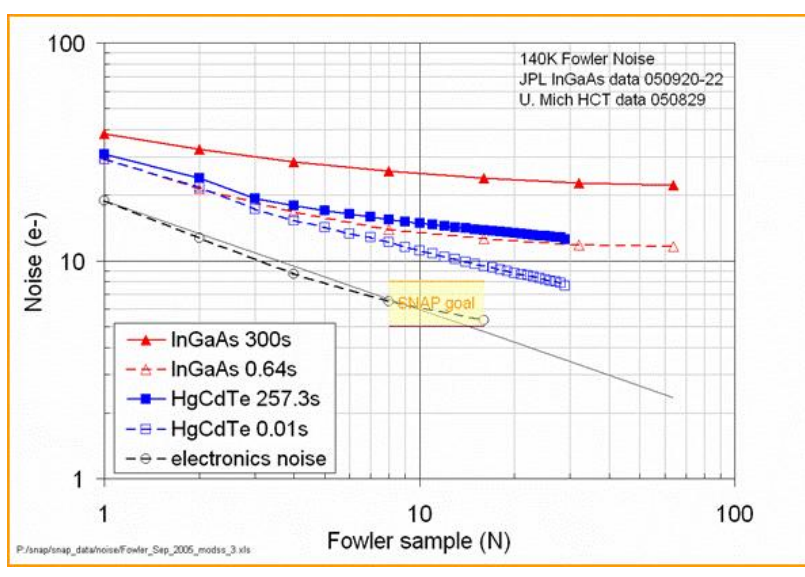

Figure 9: Comparison of the noise performance of HCT and $\mathrm{InGaAs}$ at $140 \mathrm{~K}$.

Figure 9 illustrates the noise performance of the InGaAs focal plane array versus sampling depth. The baseline noise is similar for the two imagers. While the HCT imager has a lower noise floor, both imagers are still detector noise limited. This can be seen by noting that the electronics noise (obtained by holding the reset switch closed during the measurement) is well behaved, decreasing in line with the expected square root dependence with increasing number of 
samples, while the excess detector noise does not. The HCT imager has $\sim 2 \mathrm{x}$ less correlated noise than the InGaAs imager.

Persistence, Figure 10, is seen to decay in the InGaAs detector to $<0.1 \%$ of the original signal level almost instantaneously, with a subsequent decay fit by a single, $55 \mathrm{sec}$, time constant. This corresponds to a $0.3 \%$ persistence in an equivalent HCT FPA with a rapid $17 \mathrm{sec}$ time constant, followed by a slower 150s time constant. To first order, there are no significant differences in the general behavior of persistence in the two imagers.

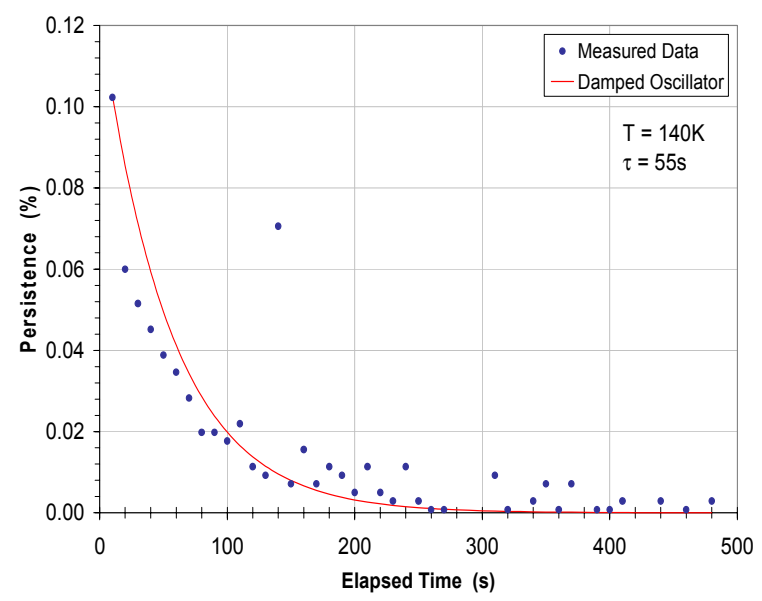

Figure 10: 140K persistence data for an InGaAs focal plane array. The initial illumination level was $\sim 80 \%$ of full well.

In conclusion, the low temperature performance of existing InGaAs detector technology is well behaved and sufficient for all but the most stringent scientific applications. The key differences observed are the magnitude of the temperaturedependent shift in cut-off wavelength and the fact that the read noise is higher than that obtained for the most recentlydeveloped HCT. Significantly, there appears to be no technological barriers towards adoption of this technology for science applications.

\section{ACKNOWLEDGEMENTS}

The effort described herein was carried out at the Jet Propulsion Laboratory, California Institute of Technology, under a contract with the National Aeronautics and Space Administration and funded through the Science \& Technology Infrastructure program. The detector development and procurement was sponsored by the United States Department of Energy under contract No. DE-AC02-05CH11231.

\section{REFERENCES}

1. M. Schubnell et al., Near infrared detectors for SNAP, Astronomical Telescopes and Instrumentation 2006, Proc. SPIE 6276 paper 29.

2. R. Smith, et al., Noise and zero-point drifts in 1.7um cutoff detectors for SNAP, Astronomical Telescopes and Instrumentation 2006, Proc. SPIE 6276 paper 30.

3. R.J. van der A et al., Low noise InGaAs infrared (1.0-2.um) focal plane arrays for SCIAMACHY, SPIE Proceedings V2957, 1997, p54.

4. T. Martin, et al., 640-512 InGaAs focal plane array camera for visible and SWIR imaging, Infrared Technology and Applications XXXI, edited by Bjørn F. Andresen,Gabor F. Fulop, Proc. of SPIE Vol. 5783 (SPIE, Bellingham, WA, $2005, \mathrm{p} 12$.

5. S. Seshadri, et. al., Characterization of NIR InGaAs imager arrays for the JDEM SNAP mission concept, Astronomical Telescopes and Instrumentation 2006, Proc. SPIE 6276 paper 31. 
6. A. Hoffman et al., Megapixel InGaAs Arrays for Low Background Applications, Infrared Technology and Applications XXXI, edited by Bjørn F. Andresen,Gabor F. Fulop, Proc. of SPIE Vol. 5783 (SPIE, Bellingham, WA, 2005, p32.

7. M.G. Brown, Development of NIR Detectors and Science Driven Requirements for SNAP, Astronomical Telescopes and Instrumentation 2006, Proc. SPIE 6265 paper 113.

8. B. Pain and B. Hancock, Accurate Measurement of Conversion Gain and Quantum Efficiency in CMOS Imagers, IS\&T/SPIE 15th. Annual Symposium, Sensors, Cameras, and Systems for Scientific/Industrial Applications Conference 5017A, January 22, 2003.

9. Autocorrelation calculation performed by M. Bonati of California Institute of Technology, Department of Astronomy, Optical Observatories group.

10. A. Moore et al., Proc. SPIE V. 5167 (2003).; A. Moore et al. QE overestimation and Deterministic Crosstalk Resulting from Interpixel Capacitance, Optical Engineering, V 45-7.; G. Finger, et al., Conversion Gain and Interpixel Capacitance of CMOS Hybrid Focal Plane Arrays, Proc. Scientific Detectors for Astronomy 2005, Eds. J.E. Beletic, P. Amico, Springer Astrophysics and Space Sciences Library, V336, 477-490.

11. V.A. Wilkinson and A.R. Adams, The effect of temperature and pressure on InGaAs band structure, Properties of lattice matched and strained InGaAs, P. Bhattacharya, ed., INSPEC EMI Series 8, 1993.

12. R. Guntupalli and R. Allen, Evaluation of InGaAs camera for scientific near infrared imaging applications, in Infrared and Photoelctronic Imagers and Detector Devices II, R.E. Longshore and A. Sood editors, Pric. SPIE V6294, (2006) p.1. 